parison with normoalbuminuric women. The variables in renal function did not differ during and after pregnancy in both groups of patients. Serum creatinine concentration and creatinine clearance showed the same kind of alterations that are generally observed in healthy pregnant women. ${ }^{5}$

The nephrotic syndrome occurred temporarily during the third trimester of pregnancy in three of the seven diabetic women with pre-existing microalbuminuria but in none of the patients with normoalbuminuria. The occurrence of the transient nephrotic syndrome in these pregnant women must be related to the pre-existing incipient diabetic nephropathy. All three patients with the nephrotic syndrome had peripheral oedema. In all cases a healthy baby was delivered by caesarean section.
In conclusion, during pregnancy a greater increase in renal protein excretion, associated with a higher risk of the transient nephrotic syndrome can be seen in diabetic women with pre-existing microalbuminuria compared with those with normoalbuminuria.

Kitzmüller J, Brown E, Philippe M, et al. Diabetic nephropathy and perinatal outcome. Am J Obstet Gynecol 1981;141:741-51.

2 Jovanovic $R$, Jovanovic $L$. Obstetric management when normoglycemia is maintained in diabetic pregnant with vascular compromise. Am f Obstet maintained in diabetic

3 Paterson L, Lunan CB, MacCuish A. Severe transient nephrotic syndrome in diabetic pregnancy. BrMed f 1985;291:1612.

+ Wallace MR, Smedley MG. The transient nephrotic syndrome of pregnancy. $N Z$ Med f 1970;71:208-12.

5 Friedberg $\mathrm{V}$. The kidney in normal pregnancy. Nieren- und HochdruckKrankheiten 1980;2:53-9.

(Accepted 27 April 1989)

\section{Uptake of influenza vaccination by patients with serious cardiac disease}

\section{J J Kurinczuk, K G Nicholson}

Infectious and Tropical

Diseases Unit, Groby Road

Hospital, Leicester

LE3 9QE

$\mathrm{J} \mathrm{J}$ Kurinczuk, MB, senior house officer in community medicine

K G Nicholson, FRCP, senior lecturer

Correspondence to: $\mathrm{Dr}$ Nicholson. required cardiac operations.
Although influenza vaccines are poor at preventing infection, they reduce the incidence of bronchopneumonia, hospital admissions, and mortality in those most at risk.' The Department of Health recommends annual vaccination for patients, especially elderly patients, with chronic pulmonary, cardiac, or renal disease; those with endocrine diseases such as diabetes; and those who are immunosuppressed.' Little is known, however, about the extent of vaccination among these target groups. We report results of a survey of influenza vaccination in patients who

\section{Patients, methods, and results}

We sent a postal questionnaire to the 251 patients aged 45 and over who were waiting for a cardiac operation and 349 randomly selected patients of similar age who had had a cardiac operation within the previous two years. Completed questionnaires were received from 565 (94\%) people; 14 people had died, and only 21 questionnaires were not returned. A concurrent review of medical notes provided additional medical details about 437 patients.

Altogether 145 of the respondents had been offered vaccination in the past, and 122 had accepted it. Thirty three had requested a vaccination. Only 85 , however, had been vaccinated within the past five years; of these, 28 had been vaccinated only once, 19 twice, 17 three times, eight four times, and 13 annually. Fifty (12\%) of the 425 patients aged $45-64$ and $35(25 \%)$ of those aged $\geqslant 65$ had been vaccinated during the past five years. There was no significant difference in the vaccination rate between men and women. Influenza vaccine had been given to only three of 57 people who had diabetes or chronic chest, endocrine, renal, or multisystem disease in addition to their cardiac problem. Eleven $(8 \%)$ of the 138 patients who had had cardiac disease for less than two years and $50(17 \%)$ of the 299 who had had it for more than two years had been vaccinated.

The patients fell into six diagnostic categories: the proportions who had been vaccinated within the past five years were $50(15 \%)$ of the 335 with coronary artery disease; $\operatorname{six}(10 \%)$ of the 63 with aortic valve disease; 15 $(19 \%)$ of the 80 with mitral valve disease; two (12\%) of the 17 with mixed valvular disease; eight $(19 \%)$ of the
41 with both coronary artery disease and valvular disease; and four (14\%) of the 29 with rarer lesions. Vaccinations had been offered by the general practitioner (69/145), an employer (67), nurse (4), others (3), or a hospital doctor (2). The offer had been made during a consultation in 65 cases, a general notice in 42 , word of mouth in 34 , and a postcard in four.

\section{Comment}

Influenza epidemics result in increased death rates, particularly in older patients and those with chronic disease or cancer. ${ }^{3}$ During such outbreaks the mortality is $0 \cdot 1 \%$ for all patients aged $\geqslant 45$ with cardiovascular disease and $0.9 \%$ for those with additional respiratory disease. Admissions to hospital and deaths associated with pneumonia and influenza can be significantly reduced by vaccination, ${ }^{4}$ and it is unfortunate that only $17 \%$ of patients who had had serious cardiac disease for over two years, for whom considerable resources are required, had been vaccinated within the previous five years. In the United States the National Institute of Allergy and Infectious Diseases reviewed more than 40 medically important diseases and ranked influenza among the top five in terms of the cost efficacy of an accelerated programme to develop vaccine.

It is encouraging that $84 \%$ of the group who had been offered vaccination had accepted it and that several patients had requested it. Remarks made by many other respondents indicated the need to educate patients and professionals about the indications for and efficacy and safety of influenza vaccination. Target groups could be identified in general practice by using the age-sex and chronic disease registers, ${ }^{5}$ and offers of vaccination could be attached to repeat prescriptions for the groups most at risk. Specialist outpatient clinics also provide opportunities to educate patients.

We thank Mr J S Bailey, Mr J N Leverment, and Mr R K Firmin for access to their register of patients with cardiac disease. JJK is a Wellcome research training fellow.

1 Nicholson K. Influenza vaccination and special risk groups. In: Wood C, ed. Influenza: strategies for prevention. London: Royal Society of Medicine, 1988:34-43.

2 Department of Health and Social Security's Joint Committee on Vaccination. Immunisation against infectious disease. London: HMSO, 1988.

3 Barker WH, Mulloolly JP. Pneumonia and influenza deaths during epidemics: implications for prevention. Arch Intern Med 1982;142:85-9.

4 Barker WH, Mullooly JP. Influenza vaccination of elderly persons: reduction in pneumonia and influenza hospitalisations and deaths. FAMA 1980;244: 25479.

5 Committee on Issues and Priorities for New Vaccine Development. New vaccine development; establishing priorities. Vol I. Diseases of importance in the United States. Washington: National Academy Press, 1985.

(Accepted 29 April 1989) 